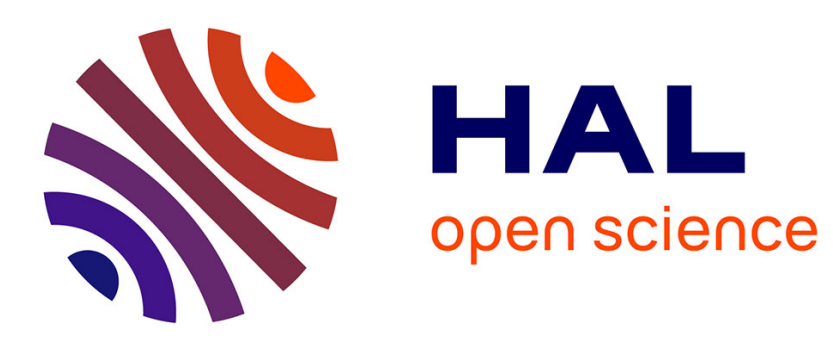

\title{
Improved Trade-based Multi-robot Coordination
}

Nicolas Jouandeau, Zhi Yan

\section{To cite this version:}

Nicolas Jouandeau, Zhi Yan. Improved Trade-based Multi-robot Coordination. 6th IEEE Joint International Information Technology and Artificial Intelligence Conference, Aug 2011, Chongqing, China. 10.1109/ITAIC.2011.6030256 . hal-02311621

\section{HAL Id: hal-02311621 \\ https://hal.science/hal-02311621}

Submitted on 11 Oct 2019

HAL is a multi-disciplinary open access archive for the deposit and dissemination of scientific research documents, whether they are published or not. The documents may come from teaching and research institutions in France or abroad, or from public or private research centers.
L'archive ouverte pluridisciplinaire HAL, est destinée au dépôt et à la diffusion de documents scientifiques de niveau recherche, publiés ou non, émanant des établissements d'enseignement et de recherche français ou étrangers, des laboratoires publics ou privés. 


\title{
Improved Trade-based Multi-robot Coordination
}

\author{
Nicolas Jouandeau Zhi Yan \\ Advanced Computing Laboratory of Saint-Denis (LIASD) \\ Paris 8 University \\ Saint-Denis 93526 France \\ $\{n, y z\} @ a i \cdot u n i v-p a r i s 8 . f r$
}

\begin{abstract}
Team work is essential to multiple mobile robot systems. An important question is, which robot should implement which action? In our previous work, we presented a trade-based task allocation approach for coordinated multirobot exploration, which simulates the relationship between buyers and sellers in a business system, to achieve dynamic task allocation by using a mechanism of unsolicited bid. This paper still addresses the problem of coordinating multi-robot exploration while presents an improved trade-based approach to raise the efficiency of task allocation by using the Hungarian method. The proposed approach has been implemented and evaluated in simulation. The experimental results demonstrate the total exploration time can be significantly reduced by the improved trade-based approach compared to previous approaches.
\end{abstract}

\section{INTRODUCTION}

Exploration of an unknown environment is a fundamental problem in robotics. It requires an agent to cover the unknown area while building a model of the environment from sensor data so as to achieve the purpose of exploration. Some exploration missions are very well known, such as the Moon exploration and the Mars exploration.

Compared with single agent, using multiple agents has a number of potential advantages [1], [2]. For example, a team of robots is able to complete an exploration mission faster than a single robot. The key to gain the advantages is coordination. Without coordination, it will not only lower mission efficiency, but also lead to the failure of the entire mission in extreme cases. An essential problem of coordination is task allocation, in other words, we need to answer such a question: which agent should execute which task [3]? Fig. 1 shows an example of multi-agent coordination. Four robots explore an unknown environment cooperatively. The result of task allocation is that different robots are responsible for exploring different rooms.

There are generally two types of mechanisms for task allocation, centralized and decentralized. The advantage of centralized mechanism is that the optimal plans can be found. Nevertheless, this mechanism is ineffectual for large teams with more agents. There is no central planner in the decentralized mechanism. Robots use locally observable informations to make their plans. This mechanism has a good adaptability and strong robustness, but the solutions it got are often sub-optimal.

The investigation context of this paper is the problem of exploring an unknown environment with a homogeneous

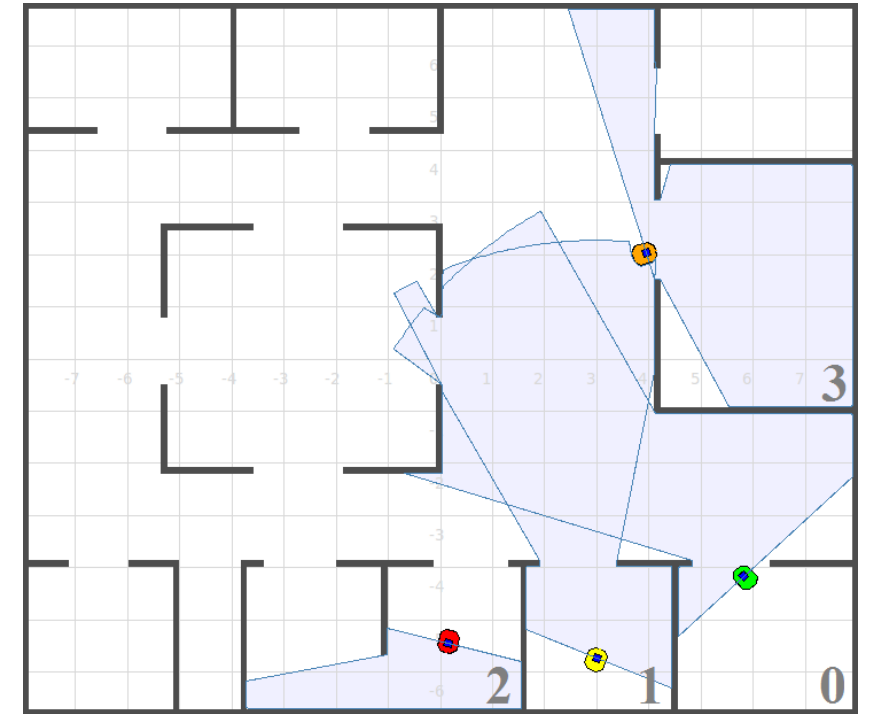

Fig. 1. Four robots explore an unknown environment cooperatively. The green robot has completed the exploration of room 0 , is moving to next room. The yellow robot is exploring room 1 . The red robot is exploring room 2 , and the orange robot is moving to room 3 .

team of cooperative robots. The objective is to explore the entire environment, while minimizing the time needed to complete the overall exploration. In our previous work [16], we developed a lightweight and robust decentralized system based on trade rules for coordinated multi-robot exploration, which is designed to simulate the relationship between buyers and sellers in a business system. The robot which plays the role of buyer makes a request for an exploration task to the robot which plays the role of seller, then the seller robot analyzes the received requests and assigns the tasks to the buyer robots reasonably. An obvious characteristic of this technique is the ability to assign multiple tasks to multiple robots at a time. Nevertheless, the previous strategy can not guarantee the global optimal task allocation plan for the whole exploration mission will be found. Therefore, in this paper, we present an improved trade-based approach for efficient task allocation, in which the buyer robot is able to make a request for several exploration tasks to the seller robot, and the latter will assign the exploration tasks to the buyer robots by using the Hungarian method [15].

The remainder of the paper is organized as follows: Section II describes an overview of some related works; 
followed by the discussion of the requirements of multirobot exploration, described in Section III; subsequently, Section IV describes our improved trade-based approach; Section V describes the experimental results obtained with our approach; the paper is concluded in Section VI.

\section{RELATED WORK}

Yamauchi [4] presented a cooperative, decentralized and fault-tolerant multi-robot exploration strategy based on the concept of frontiers. In his approach, robots share perceptual information, but maintain separate global maps. Each robot makes its own decisions about where to navigate. Whenever a robot arrives at a new frontier, it sweeps its sensors and constructs a local evidence grid representing its current surroundings. This local grid is integrated with the robots global grid, and also broadcast to all of the other robots.

Burgard et al. [5] designed a coordination component based on the approach of Yamauchi. This component applies a probabilistic method which takes the cost of reaching a frontier and its utility into account simultaneously. The cost is given by the distance of traveling to a frontier and the utility is given by the size of the unexplored area that a robot can cover from this frontier with its sensors. Whenever a frontier is assigned to a robot, the utility of the visible unexplored area of this frontier is reduced to all of the other robots.

Gerkey and Matarić [6] proposed an auction-based task allocation approach for decentralized multi-robot coordination. The auction proceeds in five steps: task announcement, metric evaluation, bid submission, close of auction and progress monitoring/contract renewal. This strategy has been implemented and tested in a general task allocation system called MURDOCH, which is built upon a principled, resource centric and publish/subscribe communication model.

Zlot et al. [7] introduced a market-based task allocation approach for multi-robot exploration. This technique borrows the market architecture which seeks to maximize benefit while minimizing cost, thus aiming to maximize utility. The benefit is information gained by visiting a goal point, the cost is the estimated distance traveled to reach the goal, then the utility is the difference between the benefit and the cost.

Besides, there are some other approaches developed with various policies [8], [9], [10], [11], [12].

\section{MULTI-ROBOT EXPLORATION}

There are two basic requirements in multi-robot exploration known as robot navigation and task allocation.

Mobile robot navigation includes three fundamental problems: map learning, localization and path planning. In a given environment, a robot should be able to determine a collision-free trajectory from its current location to a desired target location. This is known as path planning. To compute the path, a map of the environment should be known, it is built from a set of sensor data acquired by the mobile robot. The problem of map learning is commonly referred as simultaneous localization and mapping (SLAM). During the path following process, the mobile robot needs to know its exact position and orientation in the environment at all times. This is known as localization. A multi-robot navigation system should also address the possible interference between robots. For instance, a robot should take the motion of other robots into account to avoid congestion or collision [17].

Multi-robot task allocation (MRTA) should handle the problem of determining an appropriate mapping between robots and tasks. A task can be an exploration of the unknown space of environment. The key of the effective task allocation for multi-robot systems is to iterate the assignment in order to deal with changes in the tasks, the robots and the environment [3].

\section{IMPROVED TRADE-BASED APPROACH}

A business system is mainly made up of buyers and sellers. The relationship between them is known as exchange relation. Buyers can use money to purchase goods or services from sellers, then sellers collect money and sell goods or services to buyers. Our approach in this paper is built on the simulation of this relationship, the model is as follows:

$$
\text { Trade }=<R, M, T, P, C>
$$

where $R$ represents the mobile robots. $M$ represents the whole mission to be completed, which consists of several tasks, $M=\left\{m_{0}, m_{1}, \ldots, m_{n}\right\} . T$ represents the time needed (i.e. the benefit obtained) to complete the whole mission. $P$ signifies the task allocation plan. $C$ signifies the set of cost to complete the whole mission, $C=\left\{c_{0}, c_{1}, \ldots, c_{n}\right\}$. The proposed approach contains two phases which are role allocation and task allocation.

\section{A. Role allocation}

The role allocation is to solve the problem of which robot should be buyer and which robot should be seller. A simple and effective way is to number the robots in the team, and the robot which is not in task broadcast its number, then evaluates the received numbers from others after a period of time. If there is a number smaller than its own, then this robot will play the role of buyer. The details of our implementation are given in Algorithm 1.

\section{B. Task allocation}

Once the idle robot has determined its own role, it should enter the task allocation phase. For the buyer robots, the first thing to do is to choose one or more tasks to bid for and estimate the corresponding task cost. In the present study, the task is to explore an unknown region identified by topologizing the grid map of the environment [17], and the estimated task cost is the distance between the robots' current position and the target position computed by the wavefront propagation algorithm [13]:

$$
c_{r}^{m}=\text { wavefront }(\operatorname{pos}(r), \operatorname{pos}(m))
$$

where $c_{r}^{m}$ represents the estimated task cost $c$ for the robot $r$ to complete the task $m$. After completing the cost estimate, the buyer robot should send a purchase request to seller robot 


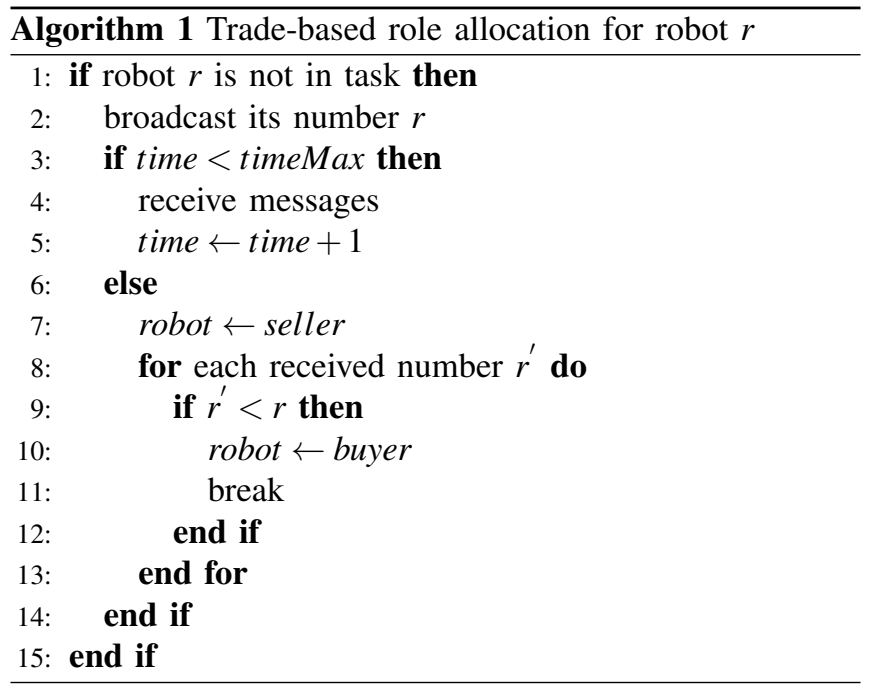

for the new task. We called this model the unsolicited bid mechanism. The purchase message contains three information:

$$
\begin{aligned}
& \text { pums }_{r}=\left\{r, M^{\prime}, C^{\prime}\right\} \\
& M^{\prime}=\left\{m_{i}, i \in \mathbb{N}_{+}\right\}, C^{\prime}=\left\{c_{i}, i \in \mathbb{N}_{+}\right\} \\
& M^{\prime} \stackrel{\text { wavefront }}{\longrightarrow} C^{\prime}
\end{aligned}
$$

where pums $_{r}$ represents the purchase message pums sent by buyer robot $r, r$ in the message means the number of the buyer robot, $M^{\prime}$ signifies the set of identifiers of the task, and $C^{\prime}$ denotes the set of estimated task cost $c_{i}$ where $c_{i}=c_{r}^{m_{i}}$. After the bidding process, the buyer robot will receive a message on whether to get the task from the seller robot or not. If the buyer robot gains a task, it will enter into the motion planning process. Otherwise, it will return to the role allocation phase for a new task.

For the seller robot, homogeneously, the first step is to select one or more tasks and estimate the corresponding task cost (the seller robot itself is also the object of task allocation). Then it should collect the purchase message from the buyer robot within a given bidding period. After this period, the seller robot will stop receiving the purchase message and evaluate the purchase requisitions received by using the Hungarian method, then assign the tasks to each buyer robot for reasonably. The Hungarian method is a combinatorial optimization algorithm which can solve assignment problems in polynomial time $O\left(n^{3}\right)$. The implementation details can be summarized by the following four steps:

1) Construct a $n \times n$ cost matrix, $n$ is the total number of the robots which have made task request. If there are more tasks than robots or conversely, we add zero columns or rows.

2) Subtract the elements of each row by the row minimum. Afterwards, subtract the elements of each column by the column minimum.

3) Find the minimum number of lines required to cover all the zeros across rows and columns. If the number of the lines is $n$, the optimal task allocation plan can be given by selecting a combination from the modified cost matrix in such a way that the sum is zero. Otherwise, go to step 4.

4) Find the smallest element which is not covered by any of the lines, then subtract it from each uncovered element and add it to each covered element. Go back to step 3 .

Suppose the seller robot has received two purchase messages: $\left\{r_{1},\left\{m_{0}, m_{1}\right\},\left\{c_{0}, c_{1}\right\}\right\}$ and $\left\{r_{2},\left\{m_{0}\right\},\left\{c_{0}\right\}\right\}$, then the $3 \times 3$ cost matrix (include the seller itself, for example, $\left.\left\{r_{0},\left\{m_{1}\right\},\left\{c_{1}\right\}\right\}\right)$ could be represented below:

$$
A=\left(\begin{array}{ccc}
\infty & r_{0}\left(c_{1}\right) & 0 \\
r_{1}\left(c_{0}\right) & r_{1}\left(c_{1}\right) & 0 \\
r_{2}\left(c_{0}\right) & \infty & 0
\end{array}\right)
$$

The task allocation message sent to buyer robot includes two information:

$$
\text { tams }_{r}=\{r, m\}
$$

where tams $_{r}$ represents the task allocation message tams sent by seller robot $r, r$ in the message means the number of the seller robot, $m$ signifies the identifier of the task. The robot which has completed a task will update its task list, and also broadcast to all teammates.

\section{EXPERIMENTS}

Our approach has been implemented and evaluated in Stage [14] a 2.5D multiple-robot simulator. The simulation experiments were conducted by using a group of Pioneer 2-DX robot equipped with a laser range finder which can provide 361 samples with 180 degrees field of view and a maximum range of 8 meters. Each robot can localize itself based on an abstract localization device which models the implementation of SLAM. The ratio between real-world time and simulation time is about 1:3. All experiments reported in this paper were carried out on a system with an Intel Core 2 Duo E8400 3.00GHz processor, an Intel Q43 Express chipset and two DDR2 $800 \mathrm{MHz} 1024 \mathrm{MB}$ dual channel memory.

To evaluate our trade-based approach, we compared our approach to an auction-based approach and used a different number of robots to conduct several experiments in various environments, Fig. 2 depicts two maps used in our simulation which are enclosed spaces with 14 meters long and 16 meters wide. The exploration team size is varied from two to six robots. The results of our experiments is given in Fig. 3 .

Fig. 3. shows a comparison of the exploration time between our improved trade-based approach and an auctionbased approach respectively in the map A (top) and the map B (bottom). From this it can be seen that the exploration time by using our approach is successfully reduced compared to the auction-based approach. On the one hand, the proposed approach introduced the unsolicited bid mechanism to support the ability to assign multiple tasks to multiple robots at a time. On the other hand, it integrated the Hungarian 


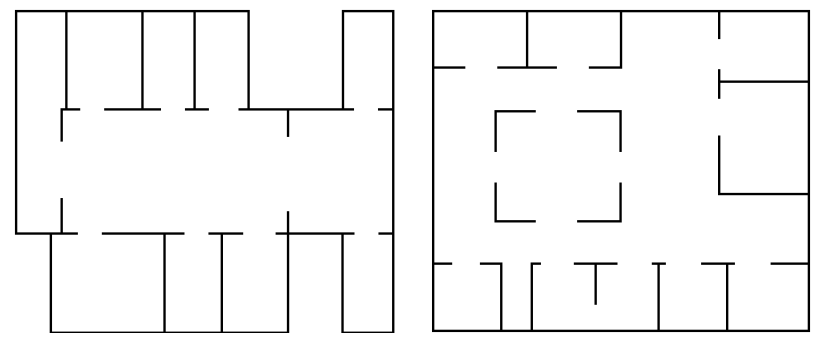

Fig. 2. Two environment maps used in our simulation: map A (left) and map B (right).
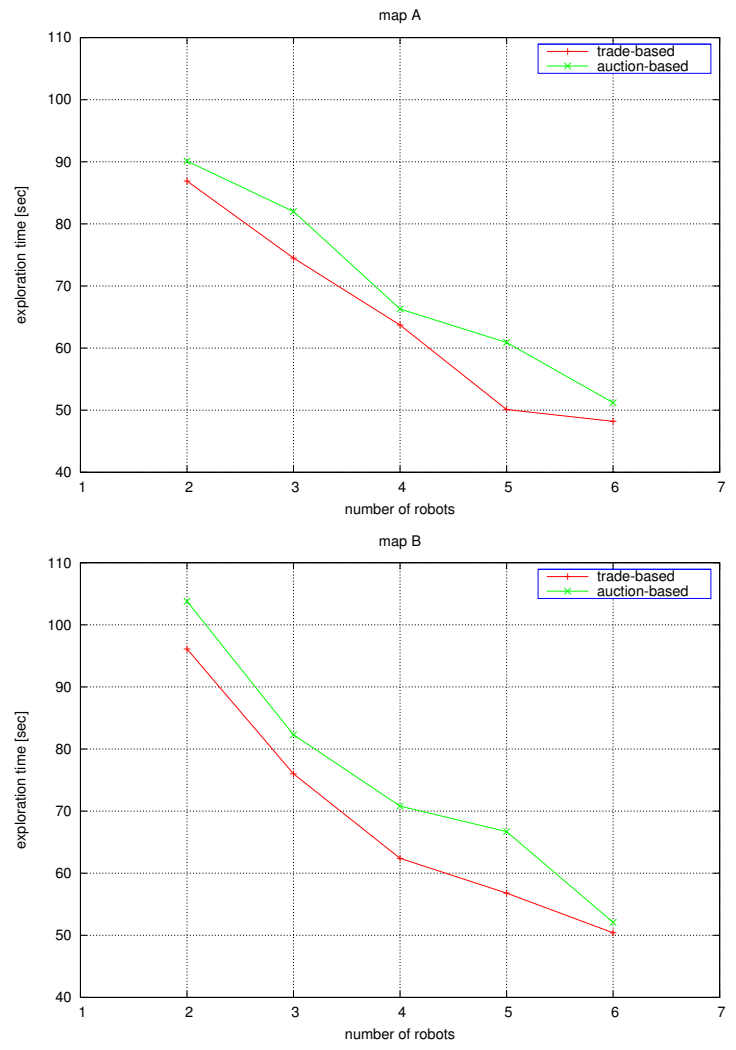

Fig. 3. Exploration time of our approach compared to an auction-based approach for the map A (top) and the map B (bottom).

method, which is an efficient method for solving assignment problems, to improve the coordinated planning of the entire multiple robot system. Moreover, due to the NP-hard of multi-robot exploration, the experimental results is an nonuniform linear dispersion.

\section{CONCLUSIONS}

In this paper, we presented an improved trade-based task allocation approach for coordinated multi-robot coordination. The trade-based method is designed to simulate the relationship between buyers and sellers in a business system, and dynamically allocate the task by using a unsolicited bid mechanism. However, the origin version can not guarantee the global optimal solution will be found, i.e. the task allocation plan for the whole mission is sometimes not optimal. This paper introduced the Hungarian method to raise the efficiency of task allocation. In contrast to the previous strategy, this version allows the buyer robot sends the purchase request to the seller robot for several tasks, then the seller robot evaluates the purchase requisitions and assign the tasks by using the Hungarian method. The experimental results demonstrate that, the time needed to accomplish the exploration mission has been significantly reduced by the improved trade-based approach.

\section{REFERENCES}

[1] G. Dudek, M. Jenkin, E. Milios, and D. Wilkes, "A taxonomy for multi-agent robotics," Autonomous Robots, vol. 3, no. 4, pp. 375-397, 1996.

[2] Y. Cao, A. Fukunaga, and A. Kahng, "Cooperative mobile robotics: Antecedents and directions," Autonomous Robots, vol. 4, no. 1, pp. 7-27, 1997.

[3] B. P. Gerkey and M. J. Matarić, "A formal analysis and taxonomy of task allocation in multi-robot systems," The International Journal of Robotics Research, vol. 23, no. 9, pp. 939-954, 2004.

[4] B. Yamauchi, "Frontier-based exploration using multiple robots," in Proceedings of the 2nd International Conference on Autonomous Agents (Agents'98), Minneapolis, MN, USA, May 1998, pp. 47-53.

[5] W. Burgard, M. Moors, D. Fox, R. Simmons, and S. Thrun, "Collaborative multi-robot exploration," in Proceedings of the 2000 IEEE International Conference on Robotics and Automation (ICRA'O0), San Francisco, CA, USA, April 2000, pp. 476-481.

[6] B. P. Gerkey and M. J. Matarić, "Sold!: Auction methods for multirobot coordination," IEEE Transactions on Robotics and Automation, Special Issue on Multi-robot Systems, vol. 18, no. 5, pp. 758-768, 2002.

[7] R. Zlot, A. Stentz, M. B. Dias, and S. Thayer, "Multi-robot exploration controlled by a market economy," in Proceedings of the 2002 IEEE International Conference on Robotics and Automation (ICRA'02), Washington, DC, USA, May 2002, pp. 3016-2023.

[8] I. M. Rekleitis, G. Dudek, and E. E. Milios, "Multi-robot collaboration for robust exploration," in Proceedings of the 2000 IEEE International Conference on Robotics and Automation (ICRA'O0), San Francisco, CA, USA, April 2000, pp. 3164-3169.

[9] J. Ko, B. Stewart, D. Fox, K. Konolige, and B. Limketkai, "A practical, decision-theoretic approach to multi-robot mapping and exploration," in Proceedings of the 2003 IEEE/RSJ International Conference on Intelligent Robots and Systems (IROS'03), Las Vegas, NV, USA, October 2003, pp. 3232-3238.

[10] L. Wu, M. A. Garcia, D. Puig, and A. Sole, "Voronoi-based space partitioning for coordinated multi-robot exploration," Journal of Physical Agents, vol. 1, no. 1, pp. 37-44, 2007.

[11] C. Stachniss, O. M. Mozos, and W. Burgard, "Efficient exploration of unknown indoor environments using a team of mobile robots," Annals of Mathematics and Artificial Intelligence, vol. 52, no. 2, pp. $205 \mathrm{ff}$, 2009.

[12] B. Yamauchi, A. Schultz, and W. Adams, "Mobile robot exploration and map-building with continuous localization," in Proceedings of the 1998 IEEE International Conference on Robotics and Automation (ICRA'98), Leuven, Belgium, May 1998, pp. 3715-3720.

[13] S. M. LaValle, Planning Algorithms, Cambridge University Press, 2006.

[14] B. P. Gerkey, R. T. Vaughan, and A. Howard, "The player/stage project: Tools for multi-robot and distributed sensor systems," in Proceedings of the 11th International Conference on Advanced Robotics (ICAR'03), Coimbra, Portugal, June 2003, pp. 317-323.

[15] H. W. Kuhn. "The Hungarian method for the assignment problem," Naval Research Logistics Quarterly, vol. 2, no. 1, pp. 83-97, 1955.

[16] Z. Yan, N. Jouandeau, and A. Ali Cherif. "Multi-robot decentralized exploration using a trade-based approach," in Proceedings of the 8th International Conference on Informatics in Control, Automation and Robotics (ICINCO 2011), Noordwijkerhout, The Netherlands, July 2011, to appear.

[17] Z. Yan, N. Jouandeau, and A. Ali Cherif. "Sampling-based multi-robot exploration," in Proceedings of the Joint 41th International Symposium on Robotics and 6th German Conference on Robotics (ISR/ROBOTIK 2010), Munich, Germany, June 2010, pp. 44-49. 